\title{
Synergies between spectroscopic and asteroseismic surveys for the Kepler field with LAMOST
}

\section{Jian-Ning Fu ${ }^{1}$, Peter De Cat $^{2}$, An-Bing Ren ${ }^{1}$, Xiao-Hu Yang ${ }^{3}$, Giovanni Catanzaro ${ }^{4}$, Christopher Corbally ${ }^{5}$, Antonio Frasca ${ }^{4}$, Richard Gray ${ }^{6}$, Joanna Molenda-Żakowicz ${ }^{7,8}$, Jian-Rong Shi ${ }^{3}$, A-Li Luo ${ }^{3}$, Hao-Tong Zhang ${ }^{3}$ and Yue $\mathrm{Wu}^{3}$}

${ }^{1}$ Department of Astronomy, Beijing Normal University, 19 Avenue Xinjiekouwai, Beijing 100875, China

email: jnfu@bnu.edu.cn

${ }^{2}$ Royal observatory of Belgium, Ringlaan 3, B-1180 Brussel, Belgium

${ }^{3}$ National Astronomical Observatories, Chinese Academy of Sciences, Beijing 100012, China

${ }^{4}$ INAF - Osservatorio Astrofisico di Catania, Via S. Sofia 78, 95123 Catania, Italy

${ }^{5}$ Vatican Observatory Research Group, Steward Observatory, Tucson, AZ 85721-0065, USA

${ }^{6}$ Department of Physics and Astronomy, Appalachian State University, Boone, NC 28608, USA

${ }^{7}$ Astronomical Institute of the University of Wrocław, ul. Kopernika 11, 51-622 Wrocław,

Poland

${ }^{8}$ Department of Astronomy, New Mexico State University, Las Cruces, NM, USA

\begin{abstract}
The NASA Kepler satellite has provided unprecedented high duty-cycle, high-precision light curves for a large number of stars by continuously monitoring a field of view in Cygnus-Lyra region, leading to great progress in both discovering exoplanets and characterizing planet-hosting stars by means of asteroseismic methods. The asteroseismic survey allows the investigation of stars covering the whole H-R diagram. However, the low precision of effective temperatures and surface gravities in the KIC catalogue and the lack of information on chemical composition, metallicity and rotation rate prevent asteroseismic modeling, requiring spectroscopic observations for thousands of asteroseismic targets in the Kepler field in a homogeneous way.
\end{abstract}

Keywords. surveys: spectroscopic; surveys: asteroseismic; astronomical missions: Kepler; astronomical facilities: LAMOST

\section{Introduction}

Asteroseismology is the only way known to probe internal structures of stars. It can be used to derive stellar parameters with unprecedented precision (radius, mass, age, etc.) and allow tests for modeling of complex dynamical processes in stellar interiors (e.g. diffusion, convective overshooting, etc.). It can also help to improve the understanding of stellar evolution. Further, the NASA space mission Kepler provides a unique opportunity for asteroseismic investigation of stars covering the whole H-R diagram (Koch et al. 2010). An extensive international collaboration was established and named as the Kepler Asteroseismic Science Consortium (KASC). As one knows, the ingredients of asteroseismology with the Kepler data include precise pulsation frequencies provided by Kepler photometry, accurately identified modes and strong constraints on atmospheric parameters to build asteroseismic models for stars. Although the Kepler Input Catalogue (KIC, Latham et al. 2005) provides $T_{\text {eff }}, \log g$ and metallicity, the precisions are in general too low for asteroseismic modeling, especially for hot and peculiar stars. In addition, the KIC lacks information on chemical composition and rotation rate of stars. 
Hence, ground-based spectroscopic observations are needed to characterize the KASC target stars.

In 2010, we initiated the LAMOST-Kepler project which aimed at collecting lowresolution spectra $(\mathrm{R}=1800)$, for as many objects from the KIC catalogue as possible, with the Large Sky Area Multi-Object Fiber Spectroscopic Telescope (LAMOST, Wang et al. 1996), a 4-m telescope equipped with 4,000 optical fibers. With the spectra, one can classify the spectral types of the stars by comparing them with the spectra of the MK secondary standard stars, derive the values of $T_{\text {eff }}, \log g$ and $[\mathrm{M} / \mathrm{H}]$ and detect chemical peculiar stars, determine the radial velocity, and detect multiple-lined systems and emission line stars. With the LAMOST-Kepler project, we plan to collect spectra for the targets and characterize them in a homogeneous way. LAMOST was the only facility able to accomplish this task in an efficient way. For more details about the project, please see De Cat et al. $(2015$.

As the largest Schmidt telescope in the world, LAMOST has an effective aperture of 4-6 meters with the Field of View (FoV) of 5deg in diameter. The light of targets is transported to 16 spectrographs through 4000 fibers. For each spectrograph, two E2V $4 \mathrm{k} \times 4 \mathrm{k}$ CCD Cameras are attached to its blue and red arms, respectively. These have spectral ranges of 370-590 $\mathrm{nm}$ and 570-900 $\mathrm{nm}$. Target selection of the LAMOST-Kepler project followed this order of priority: the $74 \mathrm{MK}$ secondary standards, the KASC targets, the 155,044 planet search group targets, and the 1,143,637 objects from the KIC catalogue. The whole FoV of the Kepler satellite was divided into 14 LAMOST-Kepler fields, at the center of each of which there is a star brighter than 8th magnitude in Johnson $V$ in order for the active optics system of LAMOST to function.

\section{Observations and Obtained Data}

In the course of 27 nights from May 30 of 2011 to September 29 of 2014, all the 14 LAMOST-Kepler fields were observed at least once with LAMOST. These observations yielded 101,086 spectra with the ratios of signal to noise (SNR) higher than 5, and they represent 80,447 individual stars. All the data were reduced with the 2-D pipeline and then 1-D pipeline of LAMOST (Luo et al. 2004). Spectra with SNR $=10$ or more, the "qualified spectra, were then fitted with both the ULySS package (Wu et al. 2011) and the ROTFIT code (Frasca et al. 2015) to estimate the stellar parameters, including the effective temperature $T_{\text {eff }}$, the surface gravity $\log g$, the metallicity $[\mathrm{Fe} / \mathrm{H}]$, and the radial velocity. Parameters for 72,086 stars were derived with ULySS. The spectra were also used to make spectral classification with the MKCLASS code (Gray et al. 2015).

\section{Parameter analysis}

The stellar parameters derived with the ULySS were compared with those available in KIC. The averaged differences of the effective temperature, the surface gravity, and the metallicity were $110 \mathrm{~K},-0.152 \mathrm{dex}$, and $0.112 \mathrm{dex}$ with the standard deviations of $495 \mathrm{~K}, 0.493 \mathrm{dex}$, and $0.376 \mathrm{dex}$, respectively. These parameters were compared with those provided by Huber et al. (2014) as well. Agreements were found for both the effective temperature and the surface gravity, but were less satisfactory for the metallicities. From the obtained parameters, 358 metal-poor stars including 53 extremely metal-poor stars were discovered. It was also found that 3 stars have absolute values of radial velocity larger than $400 \mathrm{~km} / \mathrm{s}$. 


\section{Related research work}

Some research work has already been based on the results to date of the LAMOSTKepler project. For instance, Dong et al. (2014) used 12,000 stars with LAMOST-Kepler data to compare with the KIC metallicity. It was concluded that KIC systematically underestimates both the true metallicity and the dynamic range of the Kepler sample, that the $[\mathrm{Fe} / \mathrm{H}]$ measurements of LAMOST are in good agreement with those using highresolution spectroscopy for metallicity of $[-0.3,+0.4]$ dex, and that LAMOST metallicities should be used in place of KIC metallicities whenever they are available. Deheuvels et al. (2014) provided seismic constraints on the radial dependence of the internal rotation profiles of six Kepler subgiants and young red giants. Liu et al. (2015) gave asteroseismic based estimates of the surface gravity for the LAMOST giant stars.

The data can be applied to further research work. For instance, 55906 spectra were obtained for 42209 stars for which there are Kepler observations available, which can be used to make further analysis for the stars when combined with the high-quality Kepler time-series photometric data. Spectroscopic observations of LAMOST for a large number of stars in the four open clusters in the Kepler field allow detailed study of these clusters. In addition, more than two qualified spectra were provided by LAMOST for 17,114 stars in the Kepler field, which will be helpful to detect radial velocity variable stars, etc.

\section{Conclusions and Prospects}

The first round of observation for the LAMOST-Kepler project between 2011 and 2014 has obtained 101,086 qualified spectra for 80,447 stars, leading to determination of stellar parameters for 72,086 stars. The obtained data are compared with existing databases, proven to be scientifically useful. Some research work has been made with the database.

Since May of 2015, the second round of observation of the LAMOST-Kepler project has started. More qualified data are expected for the stars in the Kepler field. Together with the Kepler photometric data and the other data for the targets in this field, more and in-depth studies could be made in multiple scientific fields, making the Kepler field a special sample of the disk of the Galaxy.

Encouraged by the progress of the LAMOST-Kepler project, the possibility of posing an "Add-on project of LAMOST for the five available K2 fields is discussed. As only around $10 \% \mathrm{~K} 2$ targets are found to have spectra in the LAMOST DR3, observations for the missing stars in the LAMOST targets would be scientifically important in the study of the stars in the five $\mathrm{K} 2$ fields.

\section{Acknowledgements}

JNF acknowledges the support from the Joint Fund of Astronomy of National Natural Science Foundation of China (NSFC) and Chinese Academy of Sciences through the Grant U1231202, and the support from the National Basic Research Program of China (973 Program 2014CB845700 and 2013CB834900). JM-Ż acknowledges the grant of the European Communitys Seventh Framework Programme (FP7/2007-2013) number 269194 and the NCN grant number 2014/13/B/ST9/00902.

\section{References}

De Cat, P., Fu, J. N., Ren, A. B., et al. 2015, ApJS, accepted

Dong, S. B., Zheng, Z., Zhu, Z., et al. 2014, ApJL, 789, L3.

Frasca, A., Molenda-Zakowicz, J., De Cat, P., et al. 2015, in preparation.

Gray, R. O., Corbally, C. J., De Cat, P., et al. 2015, in preparation. 
Huber, D., Silva Aguirre, V., Matthews, J. M., et al. 2014, ApJS, 211, 2.

Koch, D. G., Borucki, W. J., Basri, G., et al. 2010, ApJL, 713, L79

Latham, D. W., Brown, T. M., Monet, D. G., et al. 2005, in Bulletin of the American Astronomical Society, 37, American Astronomical Society Meeting Abstracts, \#110.13

Luo, A.-L., Zhang, Y.-X., \& Zhao, Y.-H., 2004, Proceedings of the SPIE, 5496, 756

Wang, S. G., Su, D. Q., Chu, Y. Q., et al. 1996, Applied Optics, 35, 5155

Wu, Y., Luo, A.-L., Li, H.-N., et al. 2011, Research in Astronomy and Astrophysics, 11, 924 